\title{
Personal preferences and discordant prostate cancer treatment choice in an intervention trial of men newly diagnosed with localized prostate cancer
}

Jaclyn LF Bosco ${ }^{*}$, Barbara Halpenny and Donna L Berry

\begin{abstract}
Background: Men diagnosed with localized prostate cancer (LPC) can choose from multiple treatment regimens and are faced with a decision in which medical factors and personal preferences are important. The Personal Patient Profile-Prostate (P3P) is a computerized decision aid for men with LPC that focuses on personal preferences. We determined whether the P3P intervention improved the concordance of treatment choice with self-reported influential side-effects compared with a control group.
\end{abstract}

Methods: English/Spanish-speaking men diagnosed with LPC (2007-2009) from four US cities were enrolled into a randomized trial and followed through 6-months via mailed or online questionnaire. Men were randomized to receive the P3P intervention or standard education plus links to reputable websites. We classified choice as concordant if men were concerned with (a) sexual function and chose external beam radiotherapy or brachytherapy, (b) bowel function and chose prostatectomy, (c) sex, bowel, and/or bladder function and chose active surveillance, or (d) not concerned with any side effect and chose any treatment. Using logistic regression, we calculated odds ratios (OR) and 95\% confidence intervals (Cl) for the association between the P3P intervention and concordance.

Results: Of 448 men, most were $<65$ years, non-Hispanic white, had multiple physician consultations prior to enrollment, and chose a treatment discordant with concerns about potential side effects. There was no significant difference in concordance between the intervention (45\%) and control (50\%) group $(\mathrm{OR}=0.82 ; 95 \% \mathrm{Cl}=0.56,1.2)$.

Conclusions: The P3P intervention did not improve concordance between potential side effects and treatment choice. Information and/or physician consultation immediately after diagnosis was likely to influence decisions despite concerns about side effects. The intervention may be more effective before the first treatment options consultation.

Trial registration: NCT00692653 http://clinicaltrials.gov/ct2/show/NCT00692653

Keywords: Localized prostate cancer, Randomized trial, Decision making, Treatment, Patient preferences, Quality of life

\footnotetext{
* Correspondence: Jaclyn_Bosco@dfci.harvard.edu

Dana-Farber Cancer Institute, 450 Brookline Avenue, LW 519, Boston, MA
} 02215, USA 


\section{Introduction}

Over 218,000 men are diagnosed with prostate cancer each year in the United States (US), of whom $90 \%$ are classified as having clinically localized prostate cancer (LPC) [1]. Men diagnosed with LPC choose from multiple treatment or surveillance regimens with the goal to increase survival and minimize treatment side effects $[2,3]$. Treatment options include radical prostatectomy, external beam radiotherapy, brachytherapy, cryosurgery, high intensity focused ultrasound (HIFU), and active surveillance (no active treatment, but PSA levels are monitored) [4]. In a prospective study of men diagnosed with LPC, sexual dysfunction (50\%) was the most common side effect one year after radical prostatectomy, while bowel dysfunction (9\%) was the most common side effect after external beam radiotherapy and brachytherapy [5]. The lack of evidence to show that one of these active treatment options results in a better survival advantage than others, except when men have higher risk disease [6], adds to the complexity of treatment decision-making for men with LPC [7-9]. Therefore, men are faced with a decision that may incorporate personal preferences based on potential side effects from certain treatments, their current health history, and lifestyle and family concerns [10].

The Decision Support Framework (DSF) [11] is organized by the 1) determinants of decisions, 2) decision support interventions, and 3) evaluation of both the process and outcomes of the decision support. While the DSF is based on expectancy value, decisional conflict and social support theories, it also addresses complex situations faced by patients in contemporary health dilemmas [11]. Deciding about treatment of LPC is one of the most appropriate decisions that fit within this framework because it encompasses careful deliberation of demographic, clinical, practice environment, perceptions of both the decision and of importance of others involved in the decision, and resources to make and implement the decision.

The Personal Patient Profile-Prostate (P3P) is a computerized, decision support system, developed under the conceptual approach to healthcare decision making using the DSF, for men with LPC that focuses on personal preferences [12]. Using data from the P3P trial, we determined whether the P3P intervention improved the concordance of treatment choice with self-reported influential side-effects compared with a control group.

\section{Methods}

\section{Study population}

A detailed description of methods from the P3P interventional trial has been reported elsewhere [12]. Briefly, men diagnosed with LPC were enrolled into a randomized trial between March 2007 and November 2009 that evaluated a computerized prostate cancer intervention used to aid in LPC treatment decision making. Men were enrolled from four geographically diverse areas in the United States. Specifically, men were enrolled from the Seattle Prostate Institute, University of Washington Seattle Cancer Care Alliance, or the Puget Sound Veteran's Affairs Hospital in Seattle, Washington, the Veteran's Affairs Hospital in San Antonio, Texas, Fox Chase Cancer Center in Philadelphia, Pennsylvania or the Veteran's Affairs Hospital in Augusta, Georgia. At the three Veteran's Affairs hospitals, men had access to all treatment options regardless of ability to pay. At all enrollment sites, eligible men were determined by their physician to be potential candidates for all treatment options. The Institutional Review Boards of the study coordinating center, the Fred Hutchinson Cancer Research Center/University of Washington Cancer Consortium, and all of enrolling sites, approved the study, and participants provided written consent.

Men diagnosed with histologically confirmed T1 or T2 prostate cancer of any risk level, which could speak and read English or Spanish at a $6^{\text {th }}$ grade level or higher, and were consulting with cancer specialists about their treatment options but had not started therapy, were eligible for the trial. All consenting participants $(\mathrm{N}=494)$ completed the Web-based Personal Patient Profile - Prostate (P3P) query component at baseline either on touch-screen computers in the clinic waiting rooms prior to the consult visit with a cancer specialist, or at home for men who had broadband Internet access. Privacy was maintained by the use of private consultation rooms or corners of waiting rooms where only the participant could view and hear the computer through privacy screens and headphones.

The P3P query component was administered at enrollment (baseline) and included measures of demographic characteristics, influential personal factors, information priorities, and current symptoms [13]. Participants were followed at 1-month and 6-months after enrollment through mailed or online questionnaires. For this analysis we evaluated the concordance between concerns about bladder function, sexual function and/or bowel function as potential treatment side effects reported at enrollment, and 6-month treatment choice; therefore men who did not return the 6-month follow-up questionnaire were not eligible for this analysis $(n=60)$.

\section{Analytic variables}

Randomization to the intervention or control group is described in detail by Berry et al. [12]. After completing the P3P query component at enrollment, participants were allocated automatically by the program to the P3P intervention software program (intervention), a computerized decision support system providing customized text and video coaching regarding the relationship between treatments and potential side effects, influential 
personal factors, and communication with health care providers [13], or standard of care plus links to reputable websites that provided basic information about prostate cancer (control). Each participant in the intervention group received brief on-screen narratives/videos plus printable teaching sheets on five topics (prognosis, stage, treatment options, side effects, sexuality) and the option to receive one or more teaching sheets on home care in terms of the impact on family and family risk of prostate cancer.

In the P3P query component, all men (intervention and control group) self-reported one of four Likert-type options for the importance or influence of potential side effects on their treatment decision ("a lot of influence", "some influence", "a little influence", or "no influence"): (a) bladder dysfunction, (b) sexual dysfunction, and/or (c) bowel dysfunction. Responses were dichotomized as "a lot" versus "some, a little and no" influence. Participants were queried about influential personal factors before indicating priority information needs and before receiving any of the customized information and coaching.

Self-reported prostate cancer treatment choice was collected at 1- and 6-months after study enrollment. Men reported choosing active surveillance, external beam radiotherapy, brachytherapy, cryosurgery, prostatectomy, HIFU, or other treatments. Participants in this trial were specifically asked about "watchful waiting", but the contemporary treatment approach of no active treatment, but monitored PSA levels is "active surveillance"; therefore, we refer to this treatment approach as "active surveillance". Those who returned the questionnaires but did not report a final treatment decision by the 6month follow-up questionnaire were classified as active surveillance. Among men who returned the 6-month follow-up questionnaire, but did not specify a treatment choice $(n=31)$, we carried forward any treatment choice previously reported at 1 -month.

Treatment choices were classified as concordant when men reported that (a) sexual function influenced their decision and they ultimately decided by 6-months to have external beam radiotherapy or brachytherapy, (b) bowel function influenced their decision and they chose radical prostatectomy, c) sexual, bowel, and/or bladder function influenced their decision and they chose active surveillance, or d) none of the concerns influenced their decision and they chose any treatment approach. Because bladder dysfunction is a side effect of both prostatectomy and radiotherapy, participants concerned with bladder function were discordant for prostatectomy as well as external beam radiotherapy and brachytherapy, but concordant with active surveillance.

\section{Potential confounding variables}

Information on socio demographic and clinical characteristics were collected at enrollment via the P3P query component and included age, weeks since biopsy, prior doctor's visit, full-time work status, college education, married/partnered, annual household income, minority race/ethnicity, and health insurance. State and trait anxiety were measured at enrollment with the Spielberger StateTrait Anxiety Inventory [14], and self-reported Expanded Prostate Index Composite-Short Form (EPIC-26) [15] was collected at enrollment and 6-months.

At enrollment, men also reported the influence that length of expected survival, other people, age, lifestyle activities, and confidence in a particular consulting physician had on their treatment choice. For each influential person (co-workers/colleagues, other friends, spouse/ partner, other family members, and famous individuals with a diagnosis of prostate cancer), we assigned a score of 0 to 3 for men who reported that their treatment decision was influenced "none", "a little", "some", and "a lot", respectively. The scores were summed across all influential people to calculate an overall "influential people score". A similar calculation was made across recreation/leisure activities and work/occupational activities. The responses for length of survival and confidence or lack of confidence in a particular doctor were also assigned scores of 0 to 3 for "none", "a little", "some", and "a lot". Men were also asked to report if age influenced their treatment decision (yes, no). No information on Gleason score, PSA levels, D'Amico risk score, or comorbidities was collected.

\section{Statistical analysis}

We described socio demographic and clinical characteristics, influential people and factors, and EPIC symptoms using frequencies and proportions for categorical variables, and means and standard deviations for continuous variables. We calculated the frequency and proportion of men whose combination of influential side effects were concordant with their treatment choice. Chi-square tests were used to determine if the proportion of concordance was significantly different between the intervention and control groups. After preliminary analysis, cryotherapy $(\mathrm{n}=3)$, HIFU $(\mathrm{n}=2)$, and other (hormonal, naturopathic, or alternative medicine) treatments $(n=7)$ were excluded from further analyses because too few men chose these treatments.

Odds ratios (OR) and 95\% confidence intervals (CI) were calculated for the association between study group and concordance using logistic regression. Confounding variables were identified a priori from our previous work (study site, age at enrollment, current work status, health insurance, Internet as an information source, trait anxiety, and the influence of people, age, recreational or work activities, confidence or lack of confidence in a particular doctor, and expected survival) [12]. We repeated the analysis stratified by treatment choice to 
assess whether the results differed by specific treatments. All analyses were conducted using SAS version 9.2 (Cary, North Carolina).

\section{Results}

After excluding 46 men due to reasons stated above, the total analytic sample included 448 men. Excluded participants did not differ by socio demographic characteristics (data not shown). The majority of the sample was nonHispanic white, currently married/partnered, received Medicare, State, or Private health insurance, used the Internet as a prostate cancer information source, and saw at least one physician prior to enrollment (Table 1). Radical prostatectomy was the most common treatment choice by 6-months (50\%).

All three concerns (bladder, sexual and bowel function) was the most commonly reported combination of side effects that influenced treatment decisions in our sample (33\%), followed by bowel function (27\%), and then no influence of any of the three potential concerns (24\%). Overall, $47 \%$ chose a treatment concordant with their concerns about influential side effects by 6-months after enrollment. There were no significant differences in the proportion of concordance between the intervention (45\%) and control $(50 \%)$ group overall $(p=0.29)$ or by specific treatment choice (Table 2). Among men who chose active surveillance $(n=68)$, concordance was $45 \%$ in the intervention and $58 \%$ in the control group $(\mathrm{p}=0.05)$, while concordance for men who chose brachytherapy $(n=63)$ was $36 \%$ in the intervention and $37 \%$ in the control group $(\mathrm{p}=0.97)$. Treatment concordance among men who chose external beam radiotherapy $(\mathrm{n}=94)$ was $32 \%$ in the intervention and $28 \%$ in the control group $(\mathrm{p}=0.65)$, and for those who chose prostatectomy $(n=223), 53 \%$ in the intervention and $56 \%$ in the control group $(\mathrm{p}=0.71)$.

As seen in Table 3, the P3P intervention group, compared with the control group, was not associated with treatment concordance overall $(\mathrm{OR}=0.82 ; 95 \% \mathrm{CI}=0.56$, 1.2) or when restricted to those who chose external beam radiotherapy $(\mathrm{OR}=1.2 ; 95 \% \mathrm{CI}=0.51,3.0)$, prostatectomy $(\mathrm{OR}=0.90 ; 95 \% \mathrm{CI}=0.53,1.5)$. None of the confounding variables substantially altered our findings (Table 3 ). The results did not change when we excluded the 31 men who skipped the treatment choice question in the 6-month follow-up questionnaire. We evaluated whether varying the classification of the potential influence on treatment choice would alter our findings, but when we collapsed "a lot" and "some" influence with "a little" and "none", the results between the intervention and concordance did not substantially change (data not shown).

\section{Discussion}

In a multi-center sample of men newly diagnosed with LPC, we observed that the majority of men who reported potential side effects of bladder, sexual, and/or bowel dysfunction as influential at enrollment, ultimately chose a treatment option within 6-months that contradicted their concerns about these side effects, regardless of receiving the P3P intervention or not.

Our results are consistent with previous studies [3,1618]. As described by Zeliadt et al., a gap between men's beliefs and knowledge regarding the efficacy and clinical evidence for prostate cancer treatment options has been identified, particularly among those who choose radical prostatectomy, which contributed to treatment choice mismatches in that study [3].

Men may minimize side effect concerns when they make a treatment decision. Side effects may be less important because men may not understand which side effect is an issue for a particular treatment option, information received is inaccurate, or the information was presented in a way that was too difficult to understand $[3,7,16,19]$. For example, Denberg et al. found that among 20 men diagnosed with LPC at a Veterans' Administration Hospital, most confused radiation therapy with chemotherapy side effects, erroneously believing hair loss to be a common side effect of radiation therapy [16]. In a qualitative study of 18 men with prostate cancer, O'Rouke found that men perceived that radical prostatectomy and external beam radiotherapy resulted in the same side effects [19]. Additionally, in a study of knowledge and understanding of LPC treatment choices among 184 men [7], over $70 \%$ of patients incorrectly answered questions related to LPC treatment complications and prognosis in the absence of treatment, and these common misperceptions were prevalent even among those who had better socioeconomic and health profiles.

Our findings indicated a high proportion of men (71\%) consulted with multiple physicians prior to enrollment. A majority of our sample may have relied on multiple consults to arrive at a treatment decision that synthesized recommendations received prior to study enrollment. This experience could explain the lack of impact for the P3P intervention on concordance. The best time to engage with a decision support system such as the P3P is likely sooner than later in the decision making process; thus implementing the intervention earlier, immediately after learning the biopsy results, could improve concordance as men are more able to consider and articulate each personal concern.

Contributing to the lack of concordance in our study was the fact that a third of our sample indicated that all three potential side effect concerns had "a lot of influence" on their treatment choice at enrollment. Participants responded to Likert-type scales to rate the importance of potential side effects. Because these men rated all of the potential side effects as equally important (tied scores) in our study, this produced a "ceiling-effect" 
Table 1 Descriptive characteristics, EPIC symptoms, and personal influences in a randomized controlled trial of men with localized prostate cancer $(\mathrm{N}=448)$

\begin{tabular}{|c|c|c|c|c|c|c|}
\hline \multirow[t]{3}{*}{ Characteristic } & \multicolumn{2}{|l|}{ Overall } & \multicolumn{2}{|c|}{ Concordant } & \multicolumn{2}{|c|}{ Not Concordant } \\
\hline & \multicolumn{2}{|l|}{$\mathrm{N}=448$} & \multicolumn{2}{|l|}{$\mathrm{N}=211$} & \multicolumn{2}{|c|}{$\mathrm{N}=237$} \\
\hline & $\mathrm{N}$ & $\%$ & $\mathrm{~N}$ & $\%$ & $\mathrm{~N}$ & $\%$ \\
\hline Intervention group & 239 & 53 & 107 & 51 & 132 & 56 \\
\hline \multicolumn{7}{|l|}{ Study site } \\
\hline Augusta, GA - Veteran's Affairs & 81 & 18 & 43 & 20 & 38 & 16 \\
\hline Philadelphia, PA - Fox Chase Cancer Center & 79 & 18 & 26 & 12 & 53 & 22 \\
\hline San Antonio, TX - Veteran's Affairs & 21 & 4.7 & 14 & 6.6 & 7 & 3.0 \\
\hline Seattle, WA - Seattle Prostate Institute & 18 & 4.0 & 10 & 4.7 & 8 & 3.4 \\
\hline University of Washington - Seattle Cancer Care Alliance & 207 & 46 & 96 & 46 & 111 & 47 \\
\hline Seattle, WA - Puget Sound Veteran's Affairs & 42 & 9.4 & 22 & 10 & 20 & 8.4 \\
\hline Age 65 or older at enrollment & 175 & 39 & 89 & 42 & 86 & 36 \\
\hline 4 or more weeks since biopsy & 297 & 66 & 141 & 67 & 156 & 66 \\
\hline Prior doctor's visit & 318 & 71 & 139 & 66 & 179 & 76 \\
\hline Minority race/ethnicity & 71 & 16 & 42 & 20 & 29 & 12 \\
\hline Currently married or partnered & 352 & 79 & 168 & 80 & 184 & 78 \\
\hline College degree or higher & 255 & 57 & 116 & 55 & 139 & 59 \\
\hline Full-time work status & 206 & 46 & 90 & 43 & 116 & 49 \\
\hline$<\$ 35,000$ annual household income & 100 & 22 & 57 & 27 & 43 & 18 \\
\hline \multicolumn{7}{|l|}{ Health Insurance } \\
\hline None or missing & 16 & 3.6 & 6 & 2.8 & 10 & 4.2 \\
\hline VA or Military & 96 & 21 & 55 & 26 & 41 & 17 \\
\hline Medicare, State, or Private & 336 & 75 & 150 & 71 & 186 & 78 \\
\hline Internet as an information source & 314 & 70 & 135 & 64 & 179 & 76 \\
\hline Influenced by age & 276 & 62 & 120 & 57 & 156 & 66 \\
\hline \multicolumn{7}{|l|}{ Treatment choice by 6-months } \\
\hline Active surveillance & 68 & 15 & 39 & 18 & 29 & 12 \\
\hline External Beam Radiotherapy & 94 & 21 & 28 & 13 & 66 & 28 \\
\hline Brachytherapy & 63 & 14 & 23 & 11 & 40 & 17 \\
\hline \multirow[t]{2}{*}{ Radical Prostatectomy } & 223 & 50 & 121 & 57 & 102 & 43 \\
\hline & Mean \pm & St Dev & Mean \pm & St Dev & Mean \pm & St Dev \\
\hline State anxiety & $40.2 \pm$ & 13.2 & $38.1 \pm$ & 12.5 & $42.0 \pm$ & 13.5 \\
\hline Trait anxiety & $33.4 \pm$ & 10.2 & $33.1 \pm$ & 9.6 & $33.6 \pm$ & 10.7 \\
\hline Baseline EPIC hormonal & $88.7 \pm$ & 12.8 & $89.1 \pm$ & 13.1 & $88.4 \pm$ & 12.6 \\
\hline Baseline EPIC urinary irritative & $84.4 \pm$ & 15.1 & $84.5 \pm$ & 13.6 & $84.2 \pm$ & 16.4 \\
\hline Baseline EPIC urinary incontinence & $92.9 \pm$ & 13.3 & $91.6 \pm$ & 14.4 & $93.9 \pm$ & 12.2 \\
\hline Baseline EPIC bowel & $94.4 \pm$ & 10.2 & $94.6 \pm$ & 10.5 & $94.2 \pm$ & 9.8 \\
\hline Baseline EPIC sexual & $64.1 \pm$ & 29.8 & $60.2 \pm$ & 31.2 & $67.5 \pm$ & 28.3 \\
\hline 6-month EPIC hormonal & $85.4 \pm$ & 16.6 & $87.0 \pm$ & 15.9 & $84.0 \pm$ & 17.2 \\
\hline 6-month EPIC urinary irritative & $82.6 \pm$ & 17.8 & $83.3 \pm$ & 17.4 & $82.0 \pm$ & 18.2 \\
\hline 6-month EPIC urinary incontinence & $73.3 \pm$ & 29.3 & $69.4 \pm$ & 30.4 & $76.8 \pm$ & 27.8 \\
\hline 6-month EPIC bowel & $90.3 \pm$ & 14.5 & $90.5 \pm$ & 14.5 & $90.1 \pm$ & 14.5 \\
\hline 6-month EPIC sexual & $40.1 \pm$ & 31.9 & $35.1 \pm$ & 30.4 & $44.6 \pm$ & 32.5 \\
\hline Index for influential people & $6.1 \pm$ & 3.0 & $5.7 \pm$ & 3.0 & $6.5 \pm$ & 2.9 \\
\hline Index of recreational and work activities & $3.9 \pm$ & 1.8 & $3.4 \pm$ & 1.9 & $4.3 \pm$ & 1.6 \\
\hline Influenced by expected survival & $2.6 \pm$ & 0.81 & $2.4 \pm$ & 0.93 & $2.7 \pm$ & 0.65 \\
\hline Influenced by confidence or lack of confidence in a particular doctor & $2.5 \pm$ & 0.90 & $2.3 \pm$ & 1.0 & $2.7 \pm$ & 0.69 \\
\hline
\end{tabular}


Table 2 Frequency and proportion of the 211 men in the P3P intervention and control group who made a concordant treatment choice by 6-months given their concerns about influential side effects (bladder, sex, and bowel) at enrollment

\begin{tabular}{|c|c|c|c|c|c|c|c|c|c|c|}
\hline \multirow{3}{*}{$\begin{array}{l}\text { Influential } \\
\text { Side Effects }\end{array}$} & \multicolumn{2}{|c|}{ Overall } & \multicolumn{2}{|c|}{ Active surveillance } & \multicolumn{2}{|l|}{$\begin{array}{l}\text { External Beam } \\
\text { Radiotherapy }\end{array}$} & \multicolumn{2}{|l|}{ Brachytherapy } & \multicolumn{2}{|c|}{$\underline{\text { Radical Prostatectomy }}$} \\
\hline & $\begin{array}{l}\text { Intervention } \\
\mathrm{N}=107\end{array}$ & $\begin{array}{l}\text { Control } \\
\mathrm{N}=104\end{array}$ & $\begin{array}{l}\text { Intervention } \\
\mathrm{N}=15\end{array}$ & $\begin{array}{l}\text { Control } \\
N=24\end{array}$ & $\begin{array}{l}\text { Intervention } \\
\mathrm{N}=15\end{array}$ & $\begin{array}{c}\text { Control } \\
N=13\end{array}$ & $\begin{array}{l}\text { Intervention } \\
\mathrm{N}=16\end{array}$ & $\begin{array}{l}\text { Control } \\
N=7\end{array}$ & $\begin{array}{l}\text { Intervention } \\
\mathrm{N}=61\end{array}$ & $\begin{array}{l}\text { Control } \\
\mathrm{N}=60\end{array}$ \\
\hline & $\mathrm{N}(\%)$ & $\mathrm{N}(\%)$ & $\mathrm{N}(\%)$ & $\mathrm{N}(\%)$ & $\mathrm{N}(\%)$ & $\mathrm{N}(\%)$ & $\mathrm{N}(\%)$ & $\mathrm{N}(\%)$ & $\mathrm{N}(\%)$ & $\mathrm{N}(\%)$ \\
\hline $\mathrm{All}^{\mathbf{a}}$ & $8(7.5)$ & $14(13)$ & $8(53)$ & $14(58)$ & $0(0)$ & $0(0)$ & $0(0)$ & $0(0)$ & $0(0)$ & $0(0)$ \\
\hline Bowel ${ }^{\mathbf{b}}$ & $31(29)$ & $32(31)$ & $0(0)$ & $0(0)$ & $0(0)$ & $0(0)$ & $0(0)$ & $0(0)$ & $31(51)$ & $32(53)$ \\
\hline Sex ${ }^{c}$ & $7(6.5)$ & $8(7.7)$ & $0(0)$ & $0(0)$ & $5(33)$ & $7(54)$ & $2(13)$ & $1(14)$ & $0(0)$ & $0(0)$ \\
\hline $\begin{array}{l}\text { Bowel and } \\
\text { Sex }\end{array}$ & $0(0)$ & $1(1.0)$ & $0(0)$ & $1(4.2)$ & $0(0)$ & $0(0)$ & $0(0)$ & $0(0)$ & $0(0)$ & $0(0)$ \\
\hline Bladder ${ }^{\mathbf{e}}$ & $1(0.9)$ & $2(1.9)$ & $1(6.7)$ & $2(8.3)$ & $0(0)$ & $0(0)$ & $0(0)$ & $0(0)$ & $0(0)$ & $0(0)$ \\
\hline None ${ }^{f}$ & $60(56)$ & $47(45)$ & $6(40)$ & $7(29)$ & $10(67)$ & $6(46)$ & $14(88)$ & $6(86)$ & $30(49)$ & $28(47)$ \\
\hline$p_{\text {-value }} \mathbf{g}$ & 0.29 & & 0.05 & & 0.65 & & 0.97 & & 0.71 & \\
\hline
\end{tabular}

a. Concerned about bladder, sexual, and bowel dysfunction concordant with active surveillance.

b. Concerned about bowel dysfunction concordant with radical prostatectomy.

c. Concerned about sexual dysfunction concordant with external beam radiotherapy and brachytherapy.

d. Concerned about bowel and sex dysfunction concordant with active surveillance.

e. Concerned about bladder dysfunction concordant with active surveillance.

f. Not concerned about any influential side effects concordant with all treatment choices.

g. p-value to test difference in concordance between intervention and control group.

and we could not discriminate the rank order. An alternative scaling method as described by Sloan et al. [20] uses Thurstone's "law of comparative judgment" to reduce the number of ties, thus avoiding the "ceiling effect" by forcing respondents to choose each of the items over each other. However, in order to implement this method, 66 paired comparisons would have been necessary because the P3P query component includes 12 information priorities: three potential side effects, and nine other influences of people (co-workers/colleagues, other friends, spouse/partner, other family members, famous individual), activities (occupational or leisure),

Table 3 Study group (intervention or control group) in relation to the concordance between influential side effects and 6-month treatment choice

\begin{tabular}{|c|c|c|c|c|c|}
\hline \multirow{2}{*}{$\begin{array}{l}\text { 6-month } \\
\text { treatment choice }\end{array}$} & \multirow{2}{*}{$\begin{array}{l}\text { Cases }^{\mathrm{a}} / \\
\text { Total }^{\mathrm{b}}\end{array}$} & \multicolumn{2}{|c|}{ Unadjusted } & \multicolumn{2}{|c|}{ Adjusted $^{c}$} \\
\hline & & OR & $95 \% \mathrm{Cl}$ & OR & $95 \% \mathrm{Cl}$ \\
\hline All treatments & $211 / 448$ & 0.82 & $(0.56,1.2)$ & 0.76 & $(0.51,1.2)$ \\
\hline Active surveillance & $39 / 68$ & 0.38 & $(0.14,1.0)$ & 0.16 & $(0.03,0.79)$ \\
\hline $\begin{array}{l}\text { External beam } \\
\text { radiotherapy }\end{array}$ & $28 / 94$ & 1.2 & $(0.51,3.0)$ & 1.3 & $(0.49,3.7)$ \\
\hline Brachytherapy & $23 / 63$ & 0.98 & $(0.32,3.0)$ & 0.96 & $(0.11,8.3)$ \\
\hline Prostatectomy & $121 / 223$ & 0.90 & $(0.53,1.5)$ & 0.87 & $(0.49,1.6)$ \\
\hline
\end{tabular}

a. Number of concordant cases.

b. Total number treated.

c. Adjusted for health care site, age at enrollment, full-time work status, health insurance type, internet as an information source for prostate cancer, trait anxiety, age as a factor in treatment choice, influence of recreational or work activities as considering treatment choice, importance or influence of confidence or lack of confidence in a particular doctor in treatment choice, and importance or influence of survival on treatment choice. confidence in clinician, and survival; thus the paired comparisons method would have been overly burdensome to our participants.

Two observational studies by Barocas et al. [21] and Cooperberg et al. [22] utilized the Cancer of the Prostate Strategic Urologic Research Endeavor (CaPSURE) registry and reported that fewer than $10 \%$ of eligible men with LPC choose active surveillance among 1,886 between 1999 and 2004 and 11,892 participants when enrollment was extended through 2008, respectively. Among men who deemed all three potential side effects concerns important to their treatment choice in our study, only 8 men in the intervention group and 14 men in the control group chose active surveillance by 6 -months. As reviewed by Pickles et al. [23], fewer men choose active surveillance because of increased anxiety from (a) the lack of intervening treatment, (b) uncertainty due to control loss, and (c) lack of education and support.

Radical prostatectomy was the most common treatment choice at 6-months in our study. At the time of the P3P trial, no LPC treatment approach had demonstrated any survival advantage over another. Regardless of educational level, Denberg et al. [16] found that men often have misconceptions about radical prostatectomy, including that removal of the tumor provides a substantial survival advantage over other treatment options. Denberg et al. [16] also reported that men also perceived that external beam radiotherapy was more convenient than surgery. Likewise, Diefenbach et al. [24] observed that men who chose radical prostatectomy perceived that their tumors were more serious than men who chose external beam radiotherapy. 
We studied a relatively large cohort of men from four geographically diverse locations in the US. We also enrolled men from both urology and radiation oncology clinics, and used a prospective design to quantitatively capture the influence of potential side effect concerns prior to making a treatment decision. The P3P intervention trial was powered to detect a decrease in decisional conflict among men newly diagnosed with LPC [12], not to increase concordance between concern for side effects and treatment choice. All participants in the trial were confirmed to have a clinically diagnosed stage I or II localized prostate cancer, but information on Gleason score, PSA levels, D'Amico risk score, physician assessment of options, or comorbidities were not collected.

While we assessed the influence of socio demographic and clinical characteristics, in addition to influences of people, activities, age, and expected survival, our study may be biased by potential unknown decision-making influences or unmeasured tumor characteristics. Additionally, we were unable to abstract medical records from all participants to confirm treatment actually received. Finally, given the homogeneity of the sample, the generalizability of our findings may be limited.

\section{Conclusion}

In conclusion, we observed that the majority of men with LPC made treatment decisions discordant with their concerns for potential side effects and the P3P intervention did not improve treatment concordance. Information and/or physician consultation immediately after diagnosis was likely to influence decisions despite concerns about side effects, as were other influential personal factors. The intervention may be more effective before the first treatment options consultation.

\section{Competing interest}

There are no financial disclosures or conflicts of interest from any authors.

\section{Authors' contributions}

JLFB was responsible for the conception and design, data analysis and interpretation, and manuscript writing. DLB and $\mathrm{BH}$ were responsible for the conception and design, protocol development, patient recruitment, and manuscript writing. All authors read and approved the final manuscript.

\section{Acknowledgements}

This study was supported by R01 NR009692 from the National Institute of Nursing Research and the National Cancer Institute of the National Institutes of Health. This material is the result of work supported with resources and use of facilities at the Charlie Norwood VA Medical Center, Augusta, GA; VA Puget Sound Healthcare System, Seattle, WA; and the South Texas Veterans Health Care System, San Antonio, TX, all of which approved the submission of the manuscript. The authors would like to thank the participants of the study for their time and participation. In addition, we gratefully acknowledge the contributions of the study investigators and staff.

Received: 21 February 2012 Accepted: 18 September 2012 Published: 28 September 2012

\section{References}

1. Mauger Rothenberg B, Marbella A, Belinson SE, Samson DJ, Bonnell CJ, Ziegler KM, Aronson N: Future Research Needs for Comparative Effectiveness of Treatments for Localized Prostate Cancer. Future Research Needs Paper No. 4. (Prepared by Blue Cross and Blue Shield Association, Technology Evaluation Center Evidence-based Practice Center under Contract No. 290-2007-10058-I.). Rockville, MD: Agency for Healthcare Research and Quality; 2010. Available at: www.

effectivehealthcare.ahrq.gov/reports/final.cfm.

2. Holmboe ES, Concato J: Treatment decisions for localized prostate cancer: asking men what's important. J Gen Intern Med 2000, 15:694-701.

3. Zeliadt SB, Ramsey SD, Penson DF, Hall IJ, Ekwueme DU, Stroud L, Lee JW: Why do men choose one treatment over another? Cancer 2006, 106 (9):1865-1874.

4. Branney P, White A, Jain S, Hiley C, Flowers P: Choosing Health, Choosing Treatment: Patient Choice After Diagnosis of Localized Prostate Cancer. Urology 2009, 74(5):968-971.

5. Sanda MG, Dunn RL, Michalski J, Sandler HM, Northouse L, Hembroff L, Lin X, Greenfield TK, Litwin MS, Saigal CS, et al: Quality of Life and Satisfaction with Outcome among Prostate-Cancer Survivors. N Engl J Med 2008, 358 (12):1250-1261.

6. Wilt T: The VA/NCI/AHRQ CSP \#407: Prostate Cancer Intervention Versus Observation Trial (PIVOT): Main Results From a Randomized Trial Comparing Radical Prostatectomy to Watchful Waiting in Men With Clinically Localized Prostate Cancer. Washington D.C: American Urological Association (AUA) Annual Meeting: May 14-19, 2011 2011; 2011.

7. Beydoun HA, Mohan R, Beydoun MA, Davis J, Lance R, Schellhammer P: Development of a scale to assess patient misperceptions about treatment choices for localized prostate cancer. BJU Int 2010, 106(3):334-341.

8. Barry MJ: The Prostate Cancer Treatment Bazaar: Comment on "Physician Visits Prior to Treatment for Clinically Localized Prostate Cancer". Arch Intern Med 2010, 170(5):450-452.

9. Evans C, Dawe R, Carson-Stevens A, Re B, et al: Choosing Health, Choosing Treatment: Patient Choice After Diagnosis of Localized Prostate Cancer (Urology 2009;74:968-971). Urology 2010, 75(3):751-751.

10. Berry DL, Ellis WJ, Woods NF, Schwien C, Mullen KH, Yang CC: Treatment decision-making by men with localized prostate cancer: the influence of personal factors. Urol Oncol 2003, 21(2):93-100.

11. O'Connor AM, Tugwell P, Wells GA, Elmslie T, Jolly E, Hollingworth G, McPherson R, Bunn H, Graham I, Drake E: A decision aid for women considering hormone therapy after menopause: decision support framework and evaluation. Patient Educ Couns 1998, 33(3):267-279.

12. Berry DL, Halpenny B, Hong F, Wolpin S, Lober WB, Russell KJ, Ellis WJ, Govindarajulu U, Bosco J, Davison BJ, et al: The Personal Patient ProfileProstate for men with newly diagnosed localized prostate cancer: a multi-site randomized trial. Urol Oncol 2011, Dec 7, Epub ahead of print. PMID 22153756.

13. Berry DL, Halpenny BSW, Davison BJ, Ellis WJ, Lober WB, McReynolds J, Wulff $\mathrm{J}$ : Development and evaluation of the personal patient profile-prostate (P3P), a Web-based decision support system for men newly diagnosed with localized prostate cancer. J Med Internet Res 2010, 12:67.

14. Spielberger C: State-Trait Anxiety Inventory for Adults (Form Y). Palo Alto, CA: Mind Garden, Inc; 1993

15. Wei JT, Dunn RL, Litwin MS, Sandler HM, Sanda MG: Development and validation of the expanded prostate cancer index composite (EPIC) for comprehensive assessment of health-related quality of life in men with prostate cancer. Urology 2000, 56(6):899-905.

16. Denberg TD, Melhado TV, Steiner JF: Patient treatment preferences in localized prostate carcinoma: The influence of emotion, misconception, and anecdote. Cancer 2006, 107(3):620-630.

17. Sommers BD, Beard CJ, D'Amico AV, Kaplan I, Richie JP, Zeckhauser RJ: Predictors of patient preferences and treatment choices for localized prostate cancer. Cancer 2008, 113(8):2058-2067.

18. Chen RC, Clark JA, Manola J, Talcott JA: Treatment 'mismatch' in early prostate cancer. Cancer 2008, 112(1):61-68.

19. O'Rourke ME: Narrowing the options: the process of deciding on prostate cancer treatment. Cancer Invest 1999, 17:349-359.

20. Sloan JA, Doig W, Yeung A: A manual to carry out Thurstone scaling and related analytic procdedures. Manitoba Nursing Research Institute Technical Report \#11. Winnipeg, Manitoba, Canada: University of Manitoba; 1994. 
21. Barocas DA, Cowan JE, Smith JA Jr, Carroll PR: What Percentage of Patients With Newly Diagnosed Carcinoma of the Prostate are Candidates for Surveillance? An Analysis of the CaPSURE(TM) Database. J Urol 2008, 180 (4):1330-1335

22. Cooperberg MR, Broering JM, Carroll PR: Time Trends and Local Variation in Primary Treatment of Localized Prostate Cancer. J Clin Oncol 2010, 28 (7):1117-1123.

23. Pickles $T$, Ruether JD, Weir $L$, Carlson L, Jakulj F, the SCT: Psychosocial barriers to active surveillance for the management of early prostate cancer and a strategy for increased acceptance. BJU Int 2007, 100(3):544551.

24. Diefenbach MA, Dorsey J, Uzzo RG, Hanks GE, Greenberg RE, Horwitz E, Newton F, Engstrom PF: Decision-making strategies for patients with localized prostate cancer. Semin Urol Oncol 2002, 1:55-62.

doi:10.1186/1477-7525-10-123

Cite this article as: Bosco et al:: Personal preferences and discordant prostate cancer treatment choice in an intervention trial of men newly diagnosed with localized prostate cancer. Health and Quality of Life

Outcomes 2012 10:123.

\section{Submit your next manuscript to BioMed Central and take full advantage of:}

- Convenient online submission

- Thorough peer review

- No space constraints or color figure charges

- Immediate publication on acceptance

- Inclusion in PubMed, CAS, Scopus and Google Scholar

- Research which is freely available for redistribution 